\title{
European DLB consortium: diagnostic and prognostic biomarkers in dementia with Lewy bodies, a multicenter international initiative
}

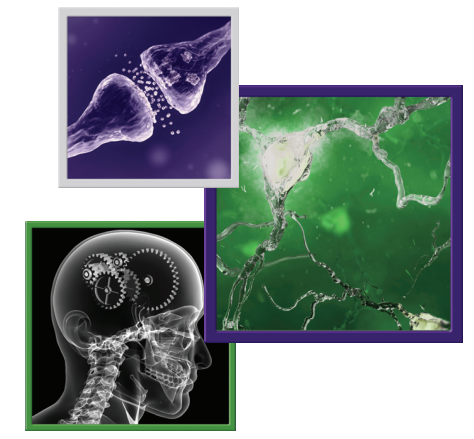

\author{
Ketil Oppedal*,1,3,4 (iD), Miguel G Borda1,2,5, Daniel Ferreira ${ }^{6}$, Eric Westman ${ }^{67}$, Dag \\ Aarsland ${ }^{18}$ \& The European DLB Consortium \\ ${ }^{1}$ Centre for Age-Related Medicine (SESAM), Stavanger University Hospital, Stavanger, Norway \\ ${ }^{2}$ Faculty of Health Sciences, University of Stavanger, Stavanger, Norway \\ ${ }^{3}$ Department of Radiology, Stavanger University Hospital, Stavanger, Norway \\ ${ }^{4}$ Department of Electrical Engineering \& Computer Science, University of Stavanger, Stavanger, Norway \\ ${ }^{5}$ Semillero de Neurociencias y Envejecimiento, Ageing Institute, Medical School, Pontificia Universidad Javeriana, Bogotá, Colombia \\ ${ }^{6}$ Division of Clinical Geriatrics, Center for Alzheimer Research, Department of Neurobiology, Care Sciences and Society, Karolinska \\ Institutet, Sweden \\ ${ }^{7}$ Department of Neuroimaging, Centre for Neuroimaging Sciences, Institute of Psychiatry, Psychology and Neuroscience, King's \\ College London, London, UK \\ ${ }^{8}$ Department of Old Age Psychiatry, Institute of Psychiatry, Psychology \& Neuroscience, King's College London, London, UK \\ *Author for correspondence: ketil.oppedal@gmail.com
}

"This project, collecting data from across Europe and the world, will make efforts to answer an urgent unmet need regarding diagnostic and prognostic markers of a disease with poor prognosis by using innovative methods and equipment”

First draft submitted: 16 August 2019; Accepted for publication: 5 September 2019; Published online: 3 October 2019

Keywords: biomarkers • dementia with Lewy bodies $\bullet$ diagnosis $\bullet$ E-DLB $\bullet$ European DLB consortium

As the population ages, the incidence of chronic diseases such as dementia increases. Dementia with Lewy bodies (DLB) is the second most common neurodegenerative dementia after Alzheimer's disease (AD) and even though both diseases are clinically separate entities [1,2], diagnosing DLB can be challenging. Despite validated diagnostic criteria for DLB [3-5], only one in three cases are correctly identified [6]. Hence, the biggest challenges in the diagnosis of DLB at present are reaching an early diagnosis and differentiating DLB from AD [7]. The current consortium diagnostic criteria for DLB have low sensitivity. Sensitivity and specificity were reported as 32 and $95 \%$, respectively against autopsy-confirmed diagnosis [8]. In addition, differential diagnosis is still a concern, particularly in early stages of the disease due to great clinical and neuropathological overlap mainly among DLB, AD and Parkinson's Disease Dementia (PDD). Reaching an accurate diagnosis is essential to personalize management to the very specific challenges DLB patients face. Besides, administering medication without a clear indication increases potential risk for patients as well as the economic burden for healthcare systems [9].

More specific biomarkers for DLB are urgently required in order to achieve better clinical therapeutic decisions. Additionally, prognostic markers are not routinely available. DLB leads to poor outcomes on key indicators including quality of life, caregiver burden, nursing home admission, hospitalization and mortality [10]. However, there is huge variability within DLB, with some patients having a very rapid decline and short survival and other patients having less rapid decline. Therefore, early discrimination between different types of patients is crucial in order to optimize management strategies and disease monitoring. There are no disease-modifying treatments available for DLB yet. However, scientific societies agree that once available, treatments should be initiated at an early disease stage, in other words, when there are still functioning neurons to salvage [11]. Early diagnosis is also crucial for clinical trials of potential novel drugs. Therefore, there is a need for identifying new biomarkers for 
improving the early detection of DLB as well as our ability to distinguish between different neurodegenerative diseases.

The European DLB consortium (E-DLB) is a multicenter and global effort concieved by leaders from the contributing centers and leading scientists in different areas of DLB research and was established in 2015. Main ambitions of the E-DLB consortium is to establish an international consensus on diagnostic criteria for DLB as well as to collect representative and high quality biomarkers data from DLB patients as part of the E-DLB study. The study has been conducted throughout an inception retrospective phase, and currently also involves the ongoing prospective phase.

\section{Retrospective E-DLB data collection}

From the E-DLB initiative, initially retrospective data from more than 1000 patients from 40 European centers with DLB data was collected, one of the largest DLB cohorts worldwide [12]. Patients were referrals from outpatient memory, movement disorders, geriatric medicine, psychiatric and neurology clinics. Local institutional ethics committee approvals were available for all centers, including the transfer of neuroimaging data. Out of this initiative, important scientific contributions have been done. Biundo et al. compared MMSE and MoCA as tools for measuring cognitive impairment on 265 patients with Lewy body diseases (LBD) and showed that both tests are comparable in measuring the rate of cognitive change over time. However, in patients with Parkinson's disease without dementia the MoCA was found to be a better measure of cognitive status as it lacks both ceiling and floor effects [13]. van Steenoven $e$ al . evaluated the prevalence of abnormal cerebrospinal fluid (CSF) AD biomarkers across the spectrum of LBD. A CSF AD profile was more common in DLB $(\mathrm{N}=375)$ compared with PDD $(\mathrm{N}=55)$ and PD $(\mathrm{N}=164)$, and it was associated with more severe cognitive impairment in DLB [14]. Abdelnour et al. reported that reduced levels of CSF amyloid $\beta$ 1-42 were associated with a more rapid cognitive decline in $\operatorname{LBD}(\mathrm{N}=100)$ [15]. Kramberger $e$ al. showed that the average annual cognitive decline measured by MMSE was approximately two points in DLB patients $(\mathrm{N}=835)$ using a linear mixed effects analyses, but this annual decline was not significantly different from that of AD or PDD patients [16]. Bonnani $e$ t al. validated quantitative electroencephalogram (EEG) as a tool for differential diagnosis between DLB $(N=79)$ and $A D(N=133)$ patients from the E-DLB cohort showing 90 and 64\% correct classification for DLB and AD, respectively. Additionally, discriminant analysis was performed to establish cutoff values for each EEG mathematical descriptor [17]. Oppedal and Ferreira et al. showed that DLB $(\mathrm{N}=333)$ patients had more overall cortical atrophy on MRI measured by visual rating scales as compared with normal controls $(\mathrm{N}=233)$ and less atrophy in the medial temporal lobe than $\mathrm{AD}(\mathrm{N}=352)$. Additionally, a signature hippocampal-sparing atrophy pattern was observed in DLB patients [18]. Morbelli et al. found that even though regions with preserved metabolism measured by fluorodeoxyglucose-positron emission tomography (FDG-PET) in 171 patients from the E-DLB cohort are relatively consistent across the DLB spectrum, core DLB features such as parkinsonism, visual hallucinations, rapid eye movement sleep behavior disorder and cognitive fluctuations were associated with hypometabolism in specific regions suggesting a close clinical-imaging correlation in DLB patients [12]. Rongve et al. reported two susceptibility loci for DLB at genomewide significance including 828 DLB patients and 82,035 normal control, highlighting the complex relationship between the genetic architecture of DLB and other neurodegenerative disorders [19]. Di Censo et al. showed that tau pathology (increased t-tau and p-tau in CSF) in 171 DLB patients was associated with less typical clinical DLB presentation with lower occurrence of Parkinsonism and lower number of concurrent core DLB features indicating a complex molecular relationship between Lewy body and AD-type pathology [20].

The common conclusion from all these published papers from the E-DLB initiative are the urgent need for future prospective studies with larger samples, centralized biomarker and neuroimaging analyses and longer follow-up leading to the prospective initiative.

\section{Prospective E-DLB data collection}

Currently, also as part of the E-DLB initiative, prospective/longitudinal data are being collected coordinated by Professor Dag Aarsland (King's College London, UK). The study is built in close collaboration with prestigious institutes not only in Europe, but also in America and Asia. One of the principal aims is to explore the utility of combinatorial biomarkers to improve diagnostic accuracy and the available methods for an early detection of the disease. Very few longitudinal studies have been reported to the present date, and no longitudinal multicenter studies with across-center harmonization of diagnostic procedures have been reported to date. 
As of today, the current consortium centers contributing to the prospective E-DLB study are: Fundació ACE (Barcelona, Spain), CMRR Strasbourg (France), Alzheimer Center (Amsterdam, The Netherlands), Memory Clinic Malmo (Malmo, Sweden), Charles University (Prague, Czech Republic), Leuven University Hospital (Leuven, Belgium), Landspitali University Hospital (Reykjavík, Iceland), University of Genoa and University of ChietiPescara (Italy), University Medical center Ljubljana, (Ljubljana, Slovenia), United Kingdom network - ENLIST study (5 centers, + more), Karolinska Institutet, Stockholm (Sweden), Stavanger University Hospital, Haugesund Hospital and Betanien Hospital in Bergen, (Norway). For a total of 623 patients with a diagnosis of probable DLB, available data are as follows: $\mathrm{MRI}=494, \mathrm{Blood}=383, \mathrm{FDG}-\mathrm{PET}=88$, Dat-scans $=159, \mathrm{CSF}=22, \mathrm{EEG}=216$ (report corresponding to July 2019).

Additionally, centers in other parts of the world have joined the initiative, in China; Kangning Hospital, Peking University Institute of Mental Health and Xuan Wu Hospital (now recruiting). Representing Latin America, Hospital Universitario San Ignacio and Fundación Valle del Lili, both centers in Colombia (waiting for protocol approvals). Collaborative studies with Mayo Clinic in United States are also ongoing [21].

This project, collecting data from across Europe and the world, will make efforts to answer an urgent unmet need regarding diagnostic and prognostic markers of a disease with poor prognosis by using innovative methods and equipment.

Many scientists from different nationalities and institutions will be working with the same cooperative data. For this reason, a global representation of a disease can be achieved, also allowing international comparisons between the participating centers. The E-DLB study is certainly novel and necessary. Findings from this global initiative may set a difference in the way we currently see, diagnose and treat DLB.

\section{Financial \& competing interests disclosure}

D Aarsland has received research support and/or honoraria from AstraZeneca, H. Lundbeck, Novartis Pharmaceuticals and GE Health, and served as paid consultant for H. Lundbeck, Eisai and Evonik. K Oppedal is funded by The Western Norway Regional Health Authority by postdoc-grant 912152. D Ferreira and E Westman are supported by the Swedish Foundation for Strategic Research (SSF); the Strategic Research Programme in Neuroscience at Karolinska Institutet (StratNeuro); the Swedish Research Council (VR); the Åke Wiberg foundation; Hjärnfonden; Alzheimerfonden; Demensfonden; and Birgitta och Sten Westerberg. The authors have no other relevant affiliations or financial involvement with any organization or entity with a financial interest in or financial conflict with the subject matter or materials discussed in the manuscript apart from those disclosed.

No writing assistance was utilized in the production of this manuscript.

\section{References}

1. Vann Jones SA, O’Brien JT. The prevalence and incidence of dementia with Lewy bodies: a systematic review of population and clinical studies. Psychol. Med. 44(4), 673-683 (2014).

2. Kane JPM, Surendranathan A, Bentley A et al. Clinical prevalence of Lewy body dementia. Alzheimers Res. Ther. 10(1), 19 (2018).

3. McKeith IG, Dickson DW, Lowe J et al. Diagnosis and management of dementia with Lewy bodies: third report of the DLB Consortium. Neurology 65(12), 1863-1872 (2005).

4. McKeith I, O'Brien J, Walker Z et al. Sensitivity and specificity of dopamine transporter imaging with 123I-FP-CIT SPECT in dementia with Lewy bodies: a Phase III, multicentre study. Lancet Neurol. 6(4), 305-313 (2007).

5. Yoshita M, Arai $\mathrm{H}$, Arai $\mathrm{H}$ et al. Diagnostic accuracy of 123I-meta-iodobenzylguanidine myocardial scintigraphy in dementia with Lewy bodies: a multicenter study. PLoS ONE 10(3), e0120540 (2015).

6. Galvin JE, Duda JE, Kaufer DI, Lippa CF, Taylor A, Zarit SH. Lewy body dementia: the caregiver experience of clinical care. Parkinsonism Relat. Disord. 16(6), 388-392 (2010).

7. Walker Z, Possin KL, Boeve BF, Aarsland D. Lewy body dementias. Lancet 386, 1683-1697 (2015).

8. Aarsland D, Rongve A, Nore SP et al. Frequency and case identification of dementia with Lewy bodies using the revised consensus criteria. Dementia Geriatric Cog. Disord. 26(5), 445-452 (2008).

9. Zhu CW, Scarmeas N, Stavitsky K et al. Comparison of costs of care between patients with Alzheimer's disease and dementia with Lewy bodies. Alzheimers Dement. 4(4), 280-284 (2008).

10. Mueller C, Ballard C, Corbett A, Aarsland D. The prognosis of dementia with Lewy bodies. Lancet Neurol. 16(5), 390-398 (2017).

11. Rizzi L, Rosset I, Roriz-Cruz M. Global epidemiology of dementia: alzheimer's and vascular types. BioMed Res. Int. 2014, 908915 (2014).

12. Morbelli S, Chincarini A, Brendel M et al. Metabolic patterns across core features in dementia with Lewy bodies. Ann. Neurol. 85(5), 715-725 (2019). 
13. Biundo R, Weis L, Bostantjopoulou S et al. MMSE and MoCA in Parkinson's disease and dementia with Lewy bodies: a multicenter 1-year follow-up study. J. Neural Transm. (Vienna) 123(4), 431-438 (2016).

14. van Steenoven I, Aarsland D, Weintraub D et al. Cerebrospinal fluid Alzheimer's disease biomarkers across the spectrum of Lewy body diseases: results from a large multicenter cohort. J. Alzheimers Dis. 54(1), 287-295 (2016).

15. Abdelnour C, van Steenoven I, Londos E et al. Alzheimer's disease cerebrospinal fluid biomarkers predict cognitive decline in lewy body dementia. Mov. Disord. 31(8), 1203-1208 (2016).

16. Kramberger MG, Auestad B, Garcia-Ptacek $S$ et al. Long-term cognitive decline in dementia with Lewy bodies in a large multicenter, international cohort. J. Alzheimers Dis. 57(3), 787-795 (2017).

17. Bonanni L, Franciotti R, Nobili F et al. EEG markers of dementia with Lewy bodies: a multicenter cohort study. J. Alzheimers Dis. 54(4), 1649-1657 (2016).

18. Oppedal K, Ferreira D, Cavallin L et al. A signature pattern of cortical atrophy in dementia with Lewy bodies: a study on 333 patients from the European DLB consortium. Alzheimers Dement. 15(3), 400-409 (2019).

19. Rongve A, Witoelar A, Ruiz A et al. GBA and APOE epsilon4 associate with sporadic dementia with Lewy bodies in European genome wide association study. Scientific Reports 9(1), 7013 (2019).

20. Di Censo R, Abdelnour C, Blanc F et al. CSF tau proteins correlate with an atypical clinical presentation in dementia with Lewy bodies. J. Neurol. Neurosurg. Psychiatry doi:10.1136/jnnp-2019-320980 (2019) (Epub ahead of print).

21. Ferreira D, Nedelska Z, Abelnour C et al. Differential impact of amyloid, tau-related pathology, and hippocampal atrophy on the clinical phenotype of DLB: a collaboration between Mayo Clinic DLB Consortium and the European DLB Consortium. Presented at: International Lewy Body Dementia Conference. Las Vegas, NV, USA, 24-26 June 2019. 